\title{
The diagnostic utility of pleural markers for tuberculosis pleural effusion
}

\author{
Man Zhang ${ }^{1}$, Dan $\mathrm{Li}^{2}$, Zhi-De Hu${ }^{3}$, Yuan-Lan Huang ${ }^{4}$ \\ ${ }^{1}$ Department of Thoracic Surgery, The Affiliated Hospital of Inner Mongolia Medical University, Hohhot 010050, China; ${ }^{2}$ Department of Special \\ Food and Equipment, Naval Special Medical Center, The Naval Military Medical University, Shanghai 200433, China; ${ }^{3}$ Department of Laboratory \\ Medicine, The Affiliated Hospital of Inner Mongolia Medical University, Hohhot 010050, China; ${ }^{4}$ Department of Laboratory Medicine, Naval \\ Special Medical Center, The Naval Military Medical University, Shanghai 200433, China \\ Contributions: (I) Conception and design: ZD Hu; (II) Administrative support: D Li; (III) Provision of study materials or patients: All authors; (IV) \\ Collection and assembly of data: M Zhang, YL Huang; (V) Data analysis and interpretation: M Zhang, ZD Hu, YL Huang; (VI) Manuscript: All \\ authors; (VII) Final approval of manuscript: All authors. \\ Correspondence to: Zhi-De Hu. Department of Laboratory Medicine, The Affiliated Hospital of Inner Mongolia Medical University, Hohhot 010050, \\ China. Email: hzdlj81@163.com; Yuan-Lan Huang. Department of Laboratory Medicine, Naval Special Medical Center, The Naval Military Medical \\ University, Shanghai 200433, China. Email: huang_yuanlan@163.com.
}

\begin{abstract}
Tuberculosis pleural effusion (TPE) is common in clinical practice, and its diagnosis remains a challenge for clinicians. Ziehl-Neelsen staining, PE Mycobacterium tuberculosis culture, and biopsy are the gold standards for TPE diagnosis; however, they are time-consuming, invasive, observer-dependent, and insensitive. PE markers represent a rapid, low-cost, and non-invasive objective diagnostic tool for TPE. In the past decades, several PE biomarkers have been developed, and their diagnostic accuracy has been evaluated in many studies. Here, we reviewed the literature to summarize the diagnostic accuracy of these biomarkers, especially using the evidence from systematic review and meta-analysis. The current research strongly suggests that adenosine deaminase (ADA), interferon-gamma (IFN- $\gamma$ ), and interleukin 27 (IL-27) have extremely higher diagnostic accuracy for TPE, while the diagnostic accuracy of interferon gamma release assays (IGRAs), tumor necrosis factor- $\alpha$ (TNF- $\alpha$ ), and interferon- $\gamma$-induced protein $10 \mathrm{kDa}$ (IP-10) is moderate. Although some evidence supports C-X-C motif chemokine ligand 9 (CXCL9), CXCL11, CXCL12, sFas ligand, angiotensin-converting enzyme (ACE), calpain-1, spectrin breakdown products (SBDP), matrix metalloproteinase-1 (MMP-1), soluble CD26 (sCD26), soluble interleukin 2 receptor (sIL-2R) as useful diagnostic markers for TPE, more support is needed to validate their diagnostic accuracy. Finally, nucleic acid amplification tests (NAATs) have extremely high diagnostic specificity, but their sensitivity is low. Taken together, ADA is the preferred marker for TPE because its low cost and suitability for standardization.
\end{abstract}

Keywords: Tuberculosis pleural effusion (TPE); diagnostic accuracy; sensitivity; specificity; pleural markers

Submitted Sep 13, 2019. Accepted for publication Sep 30, 2019.

doi: 10.21037/atm.2019.09.110

View this article at: http://dx.doi.org/10.21037/atm.2019.09.110

\section{Introduction}

Tuberculosis pleural effusion (TPE) is common in clinical practice, and separating it from other type of pleural effusion (PE), such as malignant pleural effusion (MPE), parapneumonic pleural effusion (PPE), and transudate, is often challenging (1). PE Mycobacterium tuberculosis culture, Ziehl-Neelsen staining, pleural biopsy, either guided by medical thoracoscopy, computed tomography, or ultrasound, are the gold standards for TPE diagnosis. However, these diagnostic tools have some disadvantages. PE Mycobacterium tuberculosis culture is time- and labor-consuming and thus 
does not facilitate timely diagnosis. PE Ziehl-Neelsen staining has high specificity for TPE diagnosis, but its sensitivity is unsatisfactory (2). Pleural biopsy is an invasive tool and its operation-related complications are problematic (3). Further, the accuracy of PE Ziehl-Neelsen staining and biopsy is largely affected by the skill and experience of the operator and observer.

Pleural biomarkers, in contrast to culture, staining, and biopsy, represent an inexpensive, non-invasive, rapid, and objective diagnostic tool for TPE (4). To date, many pleural biomarkers have been developed for TPE diagnosis. Here, we conducted a literature review to summarize the diagnostic accuracy of available pleural biomarkers, with particular attention paid to the results from systematic review and meta-analysis.

\section{Adenosine deaminase (ADA)}

The first report investigating ADA for TPE diagnosis was published in 1978 by Piras et al. (5). The authors enrolled 54 patients with PE (21 of them are TPE) and found that both the sensitivity and specificity of ADA were $100 \%$ at a threshold of 40 IU/L (5). Subsequently, many studies have investigated the diagnostic accuracy of ADA for TPE, but the results were varied.

In 2003, two studies systematically reviewed the diagnostic accuracy of ADA $(6,7)$ and pooled the results using meta-analysis. They reported that both the sensitivity and specificity of ADA were higher than 0.90. Notably, the method used in these two meta-analyses are based on summary receiver operating characteristic (sROC), which is not recommended in meta-analysis of diagnostic test accuracy study $(8,9)$. In 2008, a meta-analysis (10) including 63 studies with 2,796 TPE and 5,297 non-TPE cases reported that the sensitivity of specificity of ADA for TPE were 0.90 and 0.92 , respectively, with the area under sROC curve (AUC) being 0.96. An subsequent meta-analysis published in 2019 validated the meta-analysis published in 2008 (11). Another meta-analysis published in 2014 pooled the results of the studies published between 1990 and 2014 (12). A total of 12 studies were included, and the authors found that the sensitivity, specificity, and AUC of ADA were $0.86,0.88$, and 0.93 , respectively (12). The low sensitivity and specificity in this meta-analysis may be due to the fact that the literature searching strategy in this meta-analysis had some limitations. In fact, some studies included in both a later (11) and previous (10) study were missed by this meta-analysis. The researcher included 174 studies comprising 10696 TPEs 16313 non-TPEs (11) and found that the pooled sensitivity and specificity and AUC were 0.90 and 0.92 , respectively. Notably, a large portion of studies set the threshold between 30 and 50 IU/L $(7,10,11)$.

Some meta-analyses have been performed to investigate the diagnostic accuracy of ADA in Indian (13), Spanish (14), and Brazilian (15) studies. All these studies reported both a sensitivity and specificity of ADA at around 0.90 , indicating that the country source of PE has limited effects on the diagnostic accuracy of ADA.

\section{Interferon-gamma (IFN- $\gamma$ )}

The first study dealing with the diagnostic accuracy of IFN- $\gamma$ for TPE was published in 1988 (16). The authors found that pleural INF- $\gamma$ level was significantly higher than MPE, PPE, and transudate (16). Notably, sera INF- $\gamma$ was dramatically lower than that in $\mathrm{PE}$, and there were no statistical differences between TPE, MPE, PPE and transudate, indicating that pleural INF- $\gamma$ is produced locally in pleural compartment and does not arise due to the passive diffusion from sera (17). Subsequently, some studies investigated the diagnostic accuracy of pleural INF- $\gamma$ and yielded similar results (17-19).

The first meta-analysis investigating the diagnostic accuracy of IFN- $\gamma$ was published in 2003 (6). Because this meta-analysis used sROC to calculate sensitivity and specificity, other metrics such as positive and negative likelihood ratios (PLR and NLR) and diagnostic odds ratio (DOR) were not reported. In 2007, a comprehensive metaanalysis included 22 studies with $782 \mathrm{TPE}$ and 1,319 nonTPE patients (20). The pooled sensitivity and specificity of IFN- $\gamma$ were 0.89 and 0.97 , respectively. Also, the AUC was 0.98 , indicating that IFN- $\gamma$ has extremely higher diagnostic accuracy for TPE.

During the past decade, many studies have been performed to investigate the diagnostic accuracy of IFN- $\gamma$ TPE (17-19), but no updated meta-analysis has been published so far. Therefore, an updated meta-analysis is 
needed to provide new evidence.

\section{Interleukin 27 (IL-27)}

IL-27 is a member of the IL-12 family consisting of p28 and Epstein-Barr virus-induced gene protein 3 subunits (21). In 2012, Yang et al. were first to report that PF IL-27 level in TPE patients was significantly higher than that in MPE, PPE, and transudate (22). In their study, PE IL-27 had an extremely high diagnostic accuracy for TPE, with an AUC of 0.994 (22). At a threshold of $1,007 \mathrm{ng} / \mathrm{L}$, the sensitivity and specificity were 0.927 and 0.991 , respectively (22). In addition, sera IL-27 was not increased in TPE patients, indicating that high PE IL-27, like IFN- $\gamma$, is produced locally, rather than being a product of passive diffusion from sera to the pleural compartment (22). Using flow cytometry, the researchers found that pleural $\mathrm{CD} 4^{+} \mathrm{T}$ cells, $\mathrm{CD} 8^{+} \mathrm{T}$ cells, macrophages, monocytes, NKT cells, B cells, and mesothelial cells are major sources of IL-27 (22).

The high diagnostic accuracy of IL-27 has been validated by further studies $(17,23,24)$. As of now, three metaanalyses have been performed to investigate the diagnostic accuracy of PE IL-27 for TPE (25-27). All these studies have demonstrated that PE IL-27 had an AUC more than 0.95 , and both sensitivity and specificity were higher than 0.90 , also indicating that pleural IL-27 has extremely high diagnostic accuracy for TPE. In another meta-analysis, Liu et al. investigated the ability of pleural IL-27 for differentiating TPE and MPE (28). They found that the overall diagnostic accuracy of pleural IL-27 was high, with both a sensitivity and specificity of 0.97 (28).

\section{Tumor necrosis factor- $\alpha$ (TNF- $\alpha$ )}

TNF- $\alpha$ is a well-known inflammatory factor involved in infectious disease. Early research indicated that proteinpeptidoglycan complex and lipoarabinomannan have the ability to upregulate the expression of TNF- $\alpha$ in pleural fluid mononuclear cells (29). A later study reported that pleural TNF- $\alpha$ level was higher in TPE than MPE and transudates (30). This result indicates that pleural TNF- $\alpha$ is a potential diagnostic marker for TPE.

This first study investigating the diagnostic accuracy of TNF- $\alpha$ for TPE was published in 1996 (31). Subsequently, several studies have investigated the accuracy of TNF- $\alpha$ for TPE (32-34). In 2015, the first meta-analysis on the diagnostic accuracy of TNF- $\alpha$ for TPE was published (35). The authors included 7 studies and found that the sensitivity and specificity of TNF- $\alpha$ were 0.89 and 0.82 , respectively. In another meta-analysis published in 2016, the authors included 12 studies and reported a sensitivity and specificity for TNF- $\alpha$ of 0.85 and 0.80 , respectively (36). However, it should be noted that some studies also found that pleural TNF- $\alpha$ in TPE is not significantly higher than non-TPE $(37,38)$. Therefore, more evidence is needed to evaluate the diagnostic accuracy of TNF- $\alpha$.

\section{Nucleic acid amplification tests (NAATs)}

NAATs, which are based on polymerase chain reaction (PCR), have been widely used in tuberculosis diagnosis. Many types of specimens can be used in NAATs, including cerebrospinal fluid, tissue, urine, and PE. For TPE, both tissue and PE can be used for NAATs. Here, we focused on PE because of its invasiveness. NAATs are categorized as commercial or in-house tests. In 2004, a meta-analysis investigated the diagnostic accuracy of both the commercial and in-house NAATs for TPE, and found that the sensitivities were 0.62 and 0.71 , and the specificities were 0.98 and 0.93 , respectively (39). This result indicates that NAAT is useful for TPE in confirming, but not ruling out, a diagnosis.

Xpert $^{\circledR}$ MTB/RIF, a commercial NAAT, has been widely used in clinical practice. Many meta-analyses have been performed to evaluate the diagnostic accuracy of Xpert $^{\circledR}$ MTB/RIF for TPE (40-45). All of them found the specificity of Xpert ${ }^{\circledR}$ MTB/RIF to be around 0.99 , but with a sensitivity only around 0.3 and 0.5 . Notably, in two studies, the authors investigated the diagnostic accuracy of Xpert in pleural fluid versus composite reference standard (CRS), and both of them found a sensitivity around $0.20(42,43)$.

\section{Interferon gamma release assays (IGRAs)}

Lymphocytes from tuberculosis patients have previously been exposed to Mycobacterium tuberculosis, and there 
are many memory lymphocytes in circulation or in the pleural compartment. These memory lymphocytes, either in circulating or pleural effusion, are hypersensitive to mycobacterial antigen stimulation (e.g., ESAT-6, CFP-10). When stimulated with mycobacterial antigens, lymphocytes of TB infected patients release more interferon- $\gamma$ than the lymphocytes of uninfected patients (46). Therefore, interferon- $\gamma$ released by lymphocytes is an indicator of tuberculosis infection. Two types of commercially available kits have been developed, namely Quanti-FERON-TB Gold and T-SPOT-TB. The former one uses enzymelinked immunosorbent assay (ELISA) to detect interferon- $\gamma$ while the latter one uses enzyme-linked immunosorbent spot (ELISPOT).

IGRA was first developed for latent TB diagnosis, but some studies have investigated the diagnostic accuracy of IGRA for TPE. The first meta-analysis of IGRA was published in 2008 (47). The author included 8 cohorts and found that the sensitivity, specificity, and AUC of pleural IGRA were $0.75,0.82$, and 0.88 , respectively. The accuracy of blood IGRA is similar to that of pleural IGRA (47). In 2015, the meta-analysis was updated (48). The author included 16 cohorts with 516 TPE and 416 non-TPE patients and found that the sensitivity and specificity of pleural IGRA were 0.75 and 0.79 , respectively. Interestingly, another three meta-analyses published in 2015 found that the diagnostic accuracy of IGRA was high (49-51), with a sensitivity between 0.82 and 0.94 , and a specificity between 0.80 and 0.90. The AUC of the T-SPOT-TB test was higher than that of ELISA (0.98 vs. 0.84, respectively) (49). A metaanalysis only analyzed the diagnostic accuracy of T-SPOT $\mathrm{TB}$ and found that the sensitivity and specificity were 0.94 and 0.80 , respectively (50). Notably, all three metaanalyses mentioned (49-51) included a large sample size study with extremely high diagnostic accuracy (52). This may explain the different diagnostic accuracy between these meta-analyses.

\section{Soluble Fas (sFas) ligand}

In 2001, Wu et al. were first to describe significantly higher PE sFas ligand in TPE than that of MPE and transudate (53). This result was reproduced by several subsequent studies (54-56). However, the results were not reproduced by Mitani et al., who found that patients with MPE had significantly higher sFas ligand than TPE (57). None of these studies evaluated the diagnostic accuracy of sFas ligand using metrics such as sensitivity, specificity, and AUC.

In 2010, Wu et al. evaluated the diagnostic accuracy of sFas ligand in pleural effusion (58). A total of $23 \mathrm{TPE}$ and 56 non-TPE patients were enrolled, and the diagnostic performance of $\mathrm{sFas}$ ligand was evaluated by ROC curve. They found that, at a cutoff value of $39.85 \mathrm{pg} / \mathrm{mL}$, the sensitivity and specificity of sFas ligand for TPE were 0.96 and 0.80 , respectively. The AUC of sFas ligand was 0.88 , which is not significantly inferior to that of ADA $(0.91)$ and INF- $\gamma(0.91)$. Subsequently, two studies $(59,60)$ validated the findings of $\mathrm{Wu}$ et al. Both studies found that, at a cut-off value around $40 \mathrm{pg} / \mathrm{mL}$, both the sensitivity and specificity were around 0.90 , and the AUC of sFas ligand was comparable to that of ADA, IFN- $\gamma$, and interferon- $\gamma$ induced protein $10 \mathrm{kDa}$ (IP-10) (60).

\section{C-X-C motif chemokine receptor 3 (CXCR3) ligands}

CXCR3 ligands are typical IFN- $\gamma$-induced proteins, and include three chemokines: CXCL9, CXCL10, and CXCL11. CXCL10, also known as IP-10, has been extensively investigated as a diagnostic marker for TPE. In 2005, Okamoto (61) was first to report that IP-10 has high diagnostic accuracy for TPE (AUC $=0.93$ ). This result was validated by subsequent studies (19,60,62-66). In 2017, a meta-analysis investigated the diagnostic accuracy of CXCL10 for TPE (67). The authors included 14 included studies with 715 TPEs and 667 non-TPEs and found that the sensitivity and specificity of CXCL10 were 0.84 and 0.90 , respectively. The AUC under sROC of CXCL10 was 0.94 .

Thus far, only one study has investigated the diagnostic accuracy of CXCL9 and CXCL11 for TPE (68). The researchers enrolled 336 subjects with PE (106 TPE and 230 non-TPE) and simultaneously evaluated the diagnostic accuracy of ADA, IFN- $\gamma$, CXCL9, and CXCL11. They found that these four markers had comparable diagnostic accuracy, with AUCs all higher than 0.95 (68).

The diagnostic accuracy of CXCL12 was assessed in 2012; the researchers included $60 \mathrm{PE}$ patients, 15 of whom were TPE (69). At a threshold of 4,600 pg/mL, 
the diagnostic sensitivity and specificity of CXCL12 were 0.60 and 0.93 respectively. Pleural CXCL12 concentration was approximately two times higher than that in sera, indicating that CXCL12 is produced locally in the pleural compartment. However, in another study, which set MPE as the control, the diagnostic accuracy of CXCL12 was fair, with an AUC of 0.69 (70), indicating that more evidence is needed to validate the diagnostic accuracy of CXCL12.

\section{Pleural fibrosis biomarkers}

Pleural fibrosis is a common complication of TPE and occurs in approximately half the patients with TPE (71). Therefore, fibrosis-related markers are considered to be a useful diagnostic tool for TPE. In a recently published study, the researchers enrolled 47 TPE, $28 \mathrm{MPE}$, and 10 transudate patients (72). They reported that angiotensinconverting enzyme (ACE), calpain-1, spectrin breakdown products (SBDP), and matrix metalloproteinase-1 (MMP-1) were significantly higher in TPE. The AUCs of ACE, calpain, SBDP, and MMP-1 was 0.80, 0.72, 0.83, and 0.79 , respectively (72). Given that this study did not include PPE, further studies are needed to validate the findings (73). Notably, in an early study, the authors reported that rheumatoid arthritis (RA) patients have higher ACE than TPE (74), indicating that RA-induced PE should be considered for patients with higher ACE.

\section{Soluble CD26 (sCD26)}

CD26, also named dipeptidyl peptidase 4 (DPP4), is preferentially expressed on the $T$ helper. The expression of CD26 in T helper is correlated with interferon- $\gamma$ and thus represents a potential biomarker for TPE (75). There are two types of CD26, a membrane-bound form and a soluble form. In 2001, a study with a small sample size investigated the diagnostic accuracy of sCD26 for TPE (76). The authors set MPE, PPE, and heart failure as controls, and found that the sensitivity and specificity of sCD26 were 0.46 and 0.95 , respectively (76). Subsequently, three studies have investigated the diagnostic accuracy of sCD26 for TPE $(19,77,78)$. The sensitivity in these studies ranged from 0.46 to 0.87 while the specificity ranged from 0.82 to 0.95 . None of these studies reported the AUC of sCD26.

\section{Soluble interleukin 2 receptor (sIL-2R)}

Interleukin-2 (IL-2) is a cytokine that can promote the proliferation of active T-cells. Its receptor, which is expressed on the surface of activated $\mathrm{T}$ cells, can be released into circulation, which is termed sIL-2R (79). In the early 1990s, a study revealed that patients with pulmonary tuberculosis had higher sera sIL-2R compared with sarcoidosis and healthy volunteers, and anti-tuberculosis agents could decrease the sera sIL-2R level (80). Subsequent studies indicated that patients with TPE had significantly higher pleural sIL-2R than MPE, PPE, and transudate patients, and that pleural sIL-2R is positively correlated with ADA (81). These results suggest that pleural sIL-2R is a potential diagnostic marker for TPE.

In 1994, two studies investigated the diagnostic accuracy of sIL-2R TPE $(82,83)$, and both revealed that pleural sIL$2 \mathrm{R}$ is a useful diagnostic marker for TPE. Subsequently, several studies have investigated the diagnostic accuracy of sIL-2R for TPE, but the results have been varied (84-88). The sensitivity of sIL-2R ranged from 0.74 to 0.91 , and the specificity ranged from 0.31 to 1.00 . Notably, a study reported that the specificity of sIL-2R is only 0.31 (85), which is obviously different from previous studies. The reason explaining difference between these studies needs to be identified in future studies.

\section{Conclusions}

Table 1 summarizes the evidence from meta-analyses. Currently, the diagnostic accuracy of ADA, IFN- $\gamma$, IL-27, IGRA, TNF- $\alpha$, NAAT, and IP-10 has been evaluated by meta-analysis. Among the available markers, ADA, IFN- $\gamma$, and IL-27 are the most promising. Considering that ADA has advantages of low cost and suitability for standardization, we recommend ADA for TPE diagnosis. The diagnostic accuracy of IGRA, TNF- $\alpha$, and IP-10 seems inferior to that of ADA, IFN- $\gamma$, and IL-27. NAATs have extremely high diagnostic specificity, but their sensitivity is low.

Table 2 summarizes the evidence from single studies. Although some preliminary research attests to CXCL9, CXCL11, CXCL12, MMP-1, ACE, calpain-1, SBDP, sFas ligand, sCD26, and sIL-2R being useful diagnostic markers for TPE, the results are varied. Therefore, more evidence is 
Table 1 Pleural markers for tuberculosis pleural effusion: evidence from meta-analyses

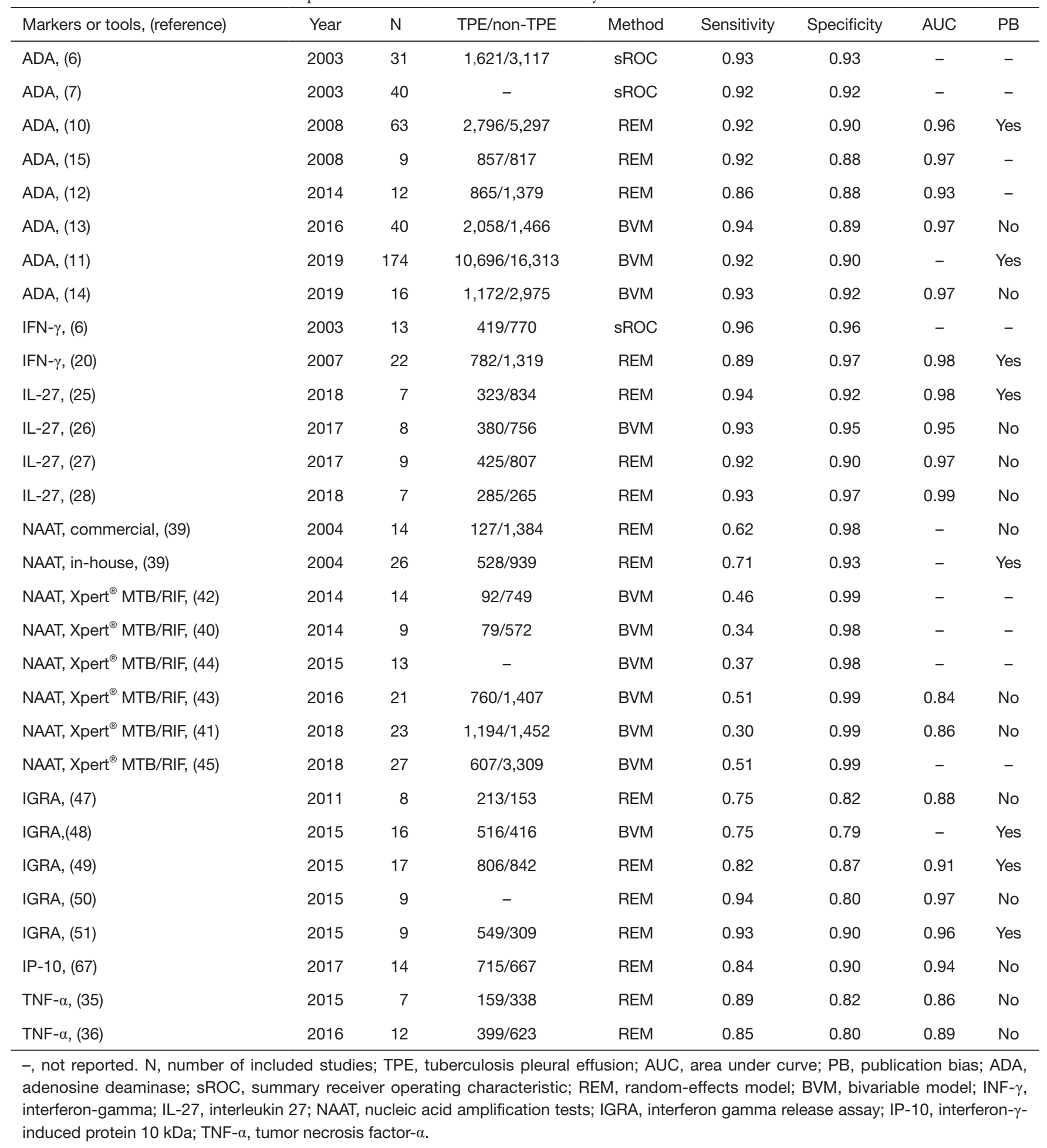


Table 2 Pleural markers for tuberculosis pleural effusion: evidence from the single studies

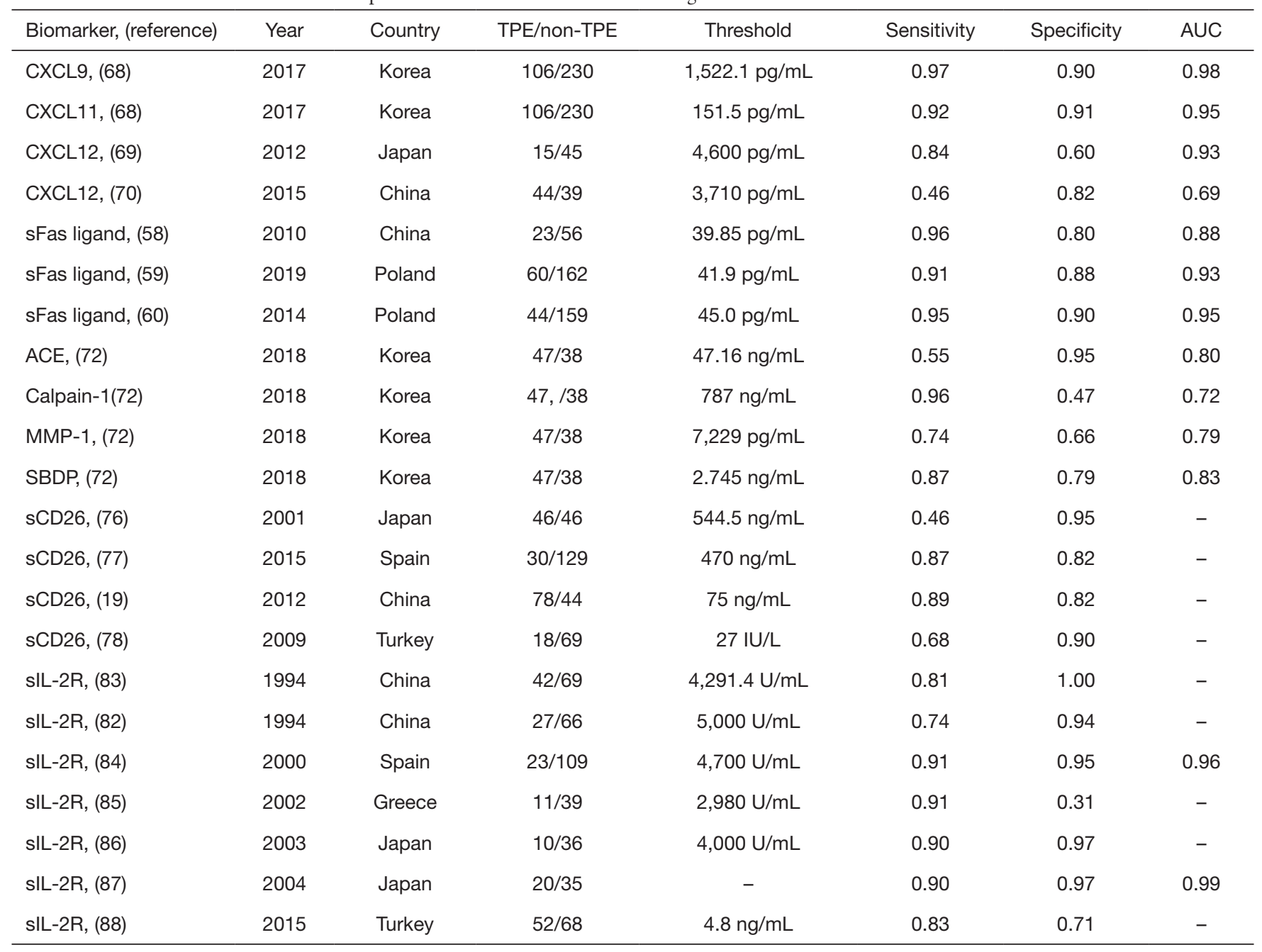

-, not reported. TPE, tuberculosis pleural effusion; AUC, area under curve; CXCL, C-X-C motif chemokine ligand; sFas, soluble Fas; ACE, angiotensin-converting enzyme; MMP-1, matrix metalloproteinase-1; SBDP, spectrin breakdown products; sCD26, soluble CD26; slL-2R, soluble interleukin 2 receptor.

needed to validate their diagnostic performance.

\section{Acknowledgements}

Funding: This study was funded by the National Natural Science Foundation of China (No. 81860501, 81601406).

\section{Footnote}

Provenance and Peer Review: This article was commissioned by the Guest Editors (Zhi-De Hu, Bing Gu) for the series "Advances in laboratory tests for infectious diseases" published in Annals of Translational Medicine. The article was sent for external peer review organized by the Guest Editors and the editorial office.

Conflicts of Interest: All authors have completed the ICMJE uniform disclosure form (available at 10.21037/ atm.2019.09.110). The series "Advances in Laboratory Tests for Infectious Diseases" was commissioned by the editorial office without any funding or sponsorship. ZDH served as the unpaid Guest Editor of the series and serves as an unpaid Executive Editor of Annals of Translational Medicine from Apr 2020 to Mar 2025. The other authors have no 
other conflicts of interest to declare.

Ethical Statement: The authors are accountable for all aspects of the work in ensuring that questions related to the accuracy or integrity of any part of the work are appropriately investigated and resolved.

Open Access Statement: This is an Open Access article distributed in accordance with the Creative Commons Attribution-NonCommercial-NoDerivs 4.0 International License (CC BY-NC-ND 4.0), which permits the noncommercial replication and distribution of the article with the strict proviso that no changes or edits are made and the original work is properly cited (including links to both the formal publication through the relevant DOI and the license). See: https://creativecommons.org/licenses/by-nc-nd/4.0/.

\section{References}

1. Zhai K, Lu Y, Shi HZ. Tuberculous pleural effusion. J Thorac Dis 2016;8:E486-94.

2. Agarwal R, Aggarwal AN, Gupta D. Diagnostic Accuracy and Safety of Semirigid Thoracoscopy in Exudative Pleural Effusions. Chest 2013;144:1857-67.

3. Wang XJ, Yang Y, Wang Z, et al. Efficacy and Safety of Diagnostic Thoracoscopy in Undiagnosed Pleural Effusions. Respiration 2015;90:251-5.

4. Porcel JM. Advances in the diagnosis of tuberculous pleuritis. Ann Transl Med 2016;4:282.

5. Piras MA, Gakis C, Budroni M, et al. Adenosine deaminase activity in pleural effusions: an aid to differential diagnosis. BMJ 1978;2:1751-2.

6. Greco S, Girardi E, Masciangelo R, et al. Adenosine deaminase and interferon gamma measurements for the diagnosis of tuberculous pleurisy: a meta-analysis. Int $\mathbf{J}$ Tuberc Lung Dis 2003;7:777-86.

7. Goto M, Noguchi $Y$, Koyama H, et al. Diagnostic value of adenosine deaminase in tuberculous pleural effusion: a meta-analysis. Ann Clin Biochem 2003;40:374-81.

8. Wang J, Leeflang M. Recommended software/packages for meta-analysis of diagnostic accuracy. J Lab Precis Med 2019;4:22.

9. Reitsma JB, Moons KG, Bossuyt PM, et al. Systematic Reviews of Studies Quantifying the Accuracy of Diagnostic Tests and Markers. Clin Chem 2012;58:1534-45.

10. Liang QL, Shi HZ, Wang K, et al. Diagnostic accuracy of adenosine deaminase in tuberculous pleurisy: A metaanalysis. Respir Med 2008;102:744-54.
11. Aggarwal AN, Agarwal R, Sehgal IS, et al. Adenosine deaminase for diagnosis of tuberculous pleural effusion: A systematic review and meta-analysis. PLoS One 2019;14:e213728.

12. Gui X, Xiao H. Diagnosis of tuberculosis pleurisy with adenosine deaminase (ADA): a systematic review and meta-analysis. Int J Clin Exp Med 2014;7:3126-35 .

13. Aggarwal AN, Agarwal R, Sehgal IS, et al. Meta-analysis of Indian studies evaluating adenosine deaminase for diagnosing tuberculous pleural effusion. Int J Tuberc Lung Dis 2016;20:1386-91.

14. Palma RM, Bielsa S, Esquerda A, et al. Diagnostic Accuracy of Pleural Fluid Adenosine Deaminase for Diagnosing Tuberculosis. Meta-analysis of Spanish Studies. Arch Bronconeumol 2019;55:23-30.

15. Morisson P, Neves DD. Evaluation of adenosine deaminase in the diagnosis of pleural tuberculosis: a Brazilian metaanalysis. J Bras Pneumol 2008;34:217-24.

16. Ribera E, Ocaña I, Martinez-Vazquez JM, et al. High Level of Interferon Gamma in Tuberculous Pleural Effusion. Chest 1988;93:308-11.

17. Wu YB, Ye ZJ, Qin SM, et al. Combined detections of interleukin 27 , interferon- $\gamma$, and adenosine deaminase in pleural effusion for diagnosis of tuberculous pleurisy. Chin Med J (Engl) 2013;126:3215-21.

18. Li M, Wang H, Wang X, et al. Diagnostic accuracy of tumor necrosis factor-alpha, interferon-gamma, interlukine- 10 and adenosine deaminase 2 in differential diagnosis between tuberculous pleural effusion and malignant pleural effusion. J Cardiothorac Surg 2014;9:118.

19. Wang H, Yue J, Yang J, et al. Clinical diagnostic utility of adenosine deaminase, interferon- $\gamma$, interferon- $\gamma$-induced protein of $10 \mathrm{kDa}$, and dipeptidyl peptidase 4 levels in tuberculous pleural effusions. Heart Lung 2012;41:70-5.

20. Jiang J, Shi HZ, Liang QL, et al. Diagnostic value of interferon-gamma in tuberculous pleurisy: a metaanalysis. Chest 2007;131:1133-41.

21. Yoshida H, Hunter CA. The Immunobiology of Interleukin-27. Annu Rev Immunol 2015;33:417-43.

22. Yang WB, Liang QL, Ye ZJ, et al. Cell origins and diagnostic accuracy of interleukin 27 in pleural effusions. PLoS One 2012;7:e40450.

23. Valdés L, San José E, Ferreiro L, et al. Interleukin 27 could be useful in the diagnosis of tuberculous pleural effusions. Respir Care 2014;59:399-405.

24. Skouras VS, Magkouta SF, Psallidas I, et al. Interleukin-27 improves the ability of adenosine deaminase to rule 
out tuberculous pleural effusion regardless of pleural tuberculosis prevalence. Infect Dis (Lond) 2015;47:477-83.

25. Wang W, Zhou Q, Zhai K, et al. Diagnostic accuracy of interleukin 27 for tuberculous pleural effusion: Two prospective studies and one meta-analysis. Thorax 2018;73:240-7.

26. Zeng N, Wan C, Qin J, et al. Diagnostic value of interleukins for tuberculous pleural effusion: a systematic review and meta-analysis. BMC Pulm Med 2017;17:180.

27. Li M, Zhu W, Khan RSU, et al. Accuracy of interleukin-27 assay for the diagnosis of tuberculous pleurisy. Medicine (Baltimore) 2017;96:e9205.

28. Liu Q, Yu YX, Wang XJ, et al. Diagnostic Accuracy of Interleukin-27 between Tuberculous Pleural Effusion and Malignant Pleural Effusion: A Meta-Analysis. Respiration 2018;95:469-77.

29. Barnes PF, Fong SJ, Brennan PJ, et al. Local production of tumor necrosis factor and IFN-gamma in tuberculous pleuritis. J Immunol 1990;145:149-54.

30. Gürsel G, Gökçora N, Elbeg S, et al. Tumor necrosis factor-alpha (TNF-alpha) in pleural fluids. Tuber Lung Dis 1995;76:370-1.

31. Orphanidou D, Gaga M, Rasidakis A, et al. Tumour necrosis factor, interleukin-1 and adenosine deaminase in tuberculous pleural effusion. Respir Med 1996;90:95-8.

32. Ogawa K, Koga H, Hirakata Y, et al. Differential diagnosis of tuberculous pleurisy by measurement of cytokine concentrations in pleural effusion. Tuber Lung Dis 1997;78:29-34.

33. Tahhan M, Ugurman F, Gozu A, et al. Tumour Necrosis Factor- $\alpha$ in Comparison to Adenosine Deaminase in Tuberculous Pleuritis. Respiration 2003;70:270-4.

34. Wong CF, Yew WW, Leung SK, et al. Assay of pleural fluid interleukin-6, tumour necrosis factor-alpha and interferongamma in the diagnosis and outcome correlation of tuberculous effusion. Respir Med 2003;97:1289-95.

35. Li Z, Qin W, Li L, et al. Diagnostic accuracy of pleural fluid tumor necrosis factor- $\alpha$ in tuberculous pleurisy: A meta-analysis. J Res Med Sci 2015;20:701.

36. Li M, Luo Z, Zhu W, et al. Diagnostic accuracy of tumor necrosis factor-alpha assay for tuberculous pleurisy. Medicine (Baltimore) 2016;95:e5510.

37. Xirouchaki N, Tzanakis N, Bouros D, et al. Diagnostic value of interleukin-1alpha, interleukin-6, and tumor necrosis factor in pleural effusions. Chest 2002;121:815-20.

38. Chomej P, Bauer K, Bitterlich N, et al. Differential diagnosis of pleural effusions by fuzzy-logic-based analysis of cytokines. Respir Med 2004;98:308-17.
39. Pai M, Flores LL, Hubbard A, et al. Nucleic acid amplification tests in the diagnosis of tuberculous pleuritis: a systematic review and meta-analysis. BMC Infect Dis 2004;4:6.

40. Maynard-Smith L, Larke N, Peters JA, et al. Diagnostic accuracy of the Xpert MTB/RIF assay for extrapulmonary and pulmonary tuberculosis when testing non-respiratory samples: a systematic review. BMC Infect Dis 2014;14:709.

41. Huo ZY, Peng L. Is Xpert MTB/RIF appropriate for diagnosing tuberculous pleurisy with pleural fluid samples? A systematic review. BMC Infect Dis 2018;18:284.

42. Denkinger CM, Schumacher SG, Boehme CC, et al. Xpert MTB/RIF assay for the diagnosis of extrapulmonary tuberculosis: a systematic review and meta-analysis. Eur Respir J 2014;44:435-46.

43. Sehgal IS, Dhooria S, Aggarwal AN, et al. Diagnostic Performance of Xpert MTB/RIF in Tuberculous Pleural Effusion: Systematic Review and Meta-analysis. J Clin Microbiol 2016;54:1133-6.

44. Penz E, Boffa J, Roberts DJ, et al. Diagnostic accuracy of the Xpert ${ }^{\circledR}$ MTB/RIF assay for extra-pulmonary tuberculosis: a meta-analysis. Int J Tuberc Lung Dis 2015;19:278-84.

45. Kohli M, Schiller I, Dendukuri N, et al. Xpert ${ }^{\circledR}$ MTB/RIF assay for extrapulmonary tuberculosis and rifampicin resistance. Cochrane Database Syst Rev 2018;8:CD012768.

46. Hooper CE, Lee YG, Maskell NA. Interferon-gamma release assays for the diagnosis of $\mathrm{TB}$ pleural effusions: hype or real hope? Curr Opin Pulm Med 2009;15:358-65.

47. Zhou Q, Chen YQ, Qin SM, et al. Diagnostic accuracy of T-cell interferon-gamma release assays in tuberculous pleurisy: a meta-analysis. Respirology 2011;16:473-80.

48. Aggarwal AN, Agarwal R, Gupta D, et al. Interferon Gamma Release Assays for Diagnosis of Pleural Tuberculosis: a Systematic Review and Meta-Analysis. J Clin Microbiol 2015;53:2451-9.

49. Pang CS, Shen YC, Tian PW, et al. Accuracy of the interferon-gamma release assay for the diagnosis of tuberculous pleurisy: an updated meta-analysis. PeerJ 2015;3:e951.

50. Zhou XX, Liu YL, Zhai K, et al. Body Fluid Interferon- $\gamma$ Release Assay for Diagnosis of Extrapulmonary Tuberculosis in Adults: A Systematic Review and MetaAnalysis. Sci Rep 2015;5:15284.

51. Li ZZ, Qin WZ, Li L, et al. Accuracy of enzyme-linked immunospot assay for diagnosis of pleural tuberculosis: a meta-analysis. Genet Mol Res 2015;14:11672-80. 
52. Liao M, Yang Q, Zhang J, et al. Gamma Interferon Immunospot Assay of Pleural Effusion Mononuclear Cells for Diagnosis of Tuberculous Pleurisy. Clin Vaccine Immunol 2014;21:347-53.

53. Wu SH, Chu JJ, Chiang CD. Increased soluble Fas ligand concentration in tuberculous pleural effusion. J Formos Med Assoc 2001;100:32-4.

54. Wang PS, Chen YM, Hsieh YL, et al. Pleural Effusion and Serum Soluble Fas-Ligand Levels Are Elevated in Different Clinical Conditions. Lung 2002;180:25-32.

55. Budak F, Uzaslan EK, Cangür Ş, et al. Increased Pleural Soluble Fas Ligand (sFasL) Levels in Tuberculosis Pleurisy and Its Relation with T-helper Type 1 Cytokines. Lung 2008;186:337-43.

56. Rajavelu P, Pokkali S, P U, et al. Comparative Evaluation of Cytokines, T-Cell Apoptosis, and Costimulatory Molecule Expression in Tuberculous and Nontuberculous Pleurisy. Clin Transl Sci 2008;1:209-14.

57. Mitani K, Nishioka Y, Yamabe K, et al. Soluble Fas in malignant pleural effusion and its expression in lung cancer cells. Cancer Sci 2003;94:302-7.

58. Wu SH, Li CTC Te, Lin CH, et al. Soluble Fas ligand is another good diagnostic marker for tuberculous pleurisy. Diagn Microbiol Infect Dis 2010;68:395-400.

59. Korczynski P, Klimiuk J, Safianowska A, et al. Impact of age on the diagnostic yield of four different biomarkers of tuberculous pleural effusion. Tuberculosis 2019;114:24-9.

60. Klimiuk J, Krenke R, Safianowska A, et al. Diagnostic Performance of Different Pleural Fluid Biomarkers in Tuberculous Pleurisy. Adv Exp Med Biol 2015;852:21-30.

61. Okamoto M, Kawabe T, Iwasaki Y, et al. Evaluation of interferon- $\gamma$, interferon- $\gamma$-inducing cytokines, and interferon- $\gamma$-inducible chemokines in tuberculous pleural effusions. J Lab Clin Med 2005;145:88-93.

62. Sutherland JS, Garba D, Fombah AE, et al. Highly Accurate Diagnosis of Pleural Tuberculosis by Immunological Analysis of the Pleural Effusion. PLoS One 2012;7:e30324.

63. Dheda K, Van-Zyl Smit RN, Sechi LA, et al. Clinical Diagnostic Utility of IP-10 and LAM Antigen Levels for the Diagnosis of Tuberculous Pleural Effusions in a High Burden Setting. PLoS One 2009;4:e4689.

64. Supriya P, Chandrasekaran P, Das SD. Diagnostic utility of interferon- $\gamma$-induced protein of $10 \mathrm{kDa}$ (IP$10)$ in tuberculous pleurisy. Diagn Microbiol Infect Dis 2008;62:186-92.

65. Santos AP, Corrêa R da S, Ribeiro-Alves M, et al. Application of Venn's diagram in the diagnosis of pleural tuberculosis using IFN- $\gamma$, IP-10 and adenosine deaminase. PLoS One 2018;13:e0202481.

66. Shu CC, Wang JY, Hsu CL, et al. Diagnostic role of inflammatory and anti-inflammatory cytokines and effector molecules of cytotoxic $\mathrm{T}$ lymphocytes in tuberculous pleural effusion. Respirology 2015;20:147-54.

67. Tong X, Lu H, Yu M, et al. Diagnostic value of interferon$\gamma$-induced protein of $10 \mathrm{kDa}$ for tuberculous pleurisy: A meta-analysis. Clin Chim Acta 2017;471:143-9.

68. Chung W, Jung Y, Lee K, et al. CXCR3 ligands in pleural fluid as markers for the diagnosis of tuberculous pleural effusion. Int J Tuberc Lung Dis 2017;21:1300-6.

69. Kohmo S, Kijima T, Mori M, et al. CXCL12 as a biological marker for the diagnosis of tuberculous pleurisy. Tuberculosis 2012;92:248-52.

70. Luo J, Wang M, Li C, et al. A novel combination of calprotectin and cxcl12 for predicting malignancy in patients with exudative pleural effusion. Medicine (Baltimore) 2015;94:e2105.

71. Meghji J, Simpson H, Squire SB, et al. A Systematic Review of the Prevalence and Pattern of Imaging Defined Post-TB Lung Disease. PLoS One 2016;11:e0161176.

72. Hong JY, Park SY, Kim Y, et al. Calpain and spectrin breakdown products as potential biomarkers in tuberculous pleural effusion. J Thorac Dis 2018;10:2558-66.

73. Zhang $M, H u$ ZD. Calpain and spectrin breakdown products in tuberculous pleural effusion. J Thorac Dis 2018;10:E654-5.

74. Söderblom T, Nyberg P, Pettersson T, et al. Pleural fluid beta-2-microglobulin and angiotensin-converting enzyme concentrations in rheumatoid arthritis and tuberculosis. Respiration 1996;63:272-6.

75. Willheim M, Ebner C, Baier K, et al. Cell surface characterization of $\mathrm{T}$ lymphocytes and allergen-specific T cell clones: Correlation of CD26 expression with T H1 subsets. J Allergy Clin Immunol 1997;100:348-55.

76. Oshikawa K, Sugiyama Y. Elevated soluble CD26 levels in patients with tuberculous pleurisy. Int J Tuberc Lung Dis 2001;5:868-72.

77. Sánchez-Otero N, Rodríguez-Berrocal FJ, de la Cadena MP, et al. Evaluation of pleural effusion sCD26 and DPPIV as diagnostic biomarkers in lung disease. Sci Rep 2014;4:3999.

78. Küpeli E, Karnak D, Elgün S, et al. Concurrent measurement of adenosine deaminase and dipeptidyl peptidase IV activity in the diagnosis of tuberculous pleural effusion. Diagn Microbiol Infect Dis 2009;65:365-71.

79. Rubin LA, Kurman CC, Fritz ME, et al. Soluble 
interleukin 2 receptors are released from activated human lymphoid cells in vitro. J Immunol 1985;135:3172-7.

80. Takahashi S, Setoguchi Y, Nukiwa T, et al. Soluble lnterleukin-2 Receptor in Sera of Patients with Pulmonary Tuberculosis. Chest 1991;99:310-4.

81. Ito M, Kojiro N, Shirasaka T, et al. Elevated Levels of Soluble Interleukin-2 Receptors in Tuberculous Pleural Effusions. Chest 1990;97:1141-3.

82. Chiang CS, Chiang CD, Lin JW, et al. Neopterin, Soluble Interleukin-2 Receptor and Adenosine Deaminase Levels in Pleural Effusions. Respiration 1994;61:150-4.

83. Chang SC, Hsu YT, Chen YC, et al. Usefulness of soluble interleukin 2 receptor in differentiating tuberculous and carcinomatous pleural effusions. Arch Intern Med 1994;154:1097-101.

84. Porcel JM, Gázquez I, Vives M, et al. Diagnosis of tuberculous pleuritis by the measurement of soluble

Cite this article as: Zhang M, Li D, Hu ZD, Huang YL. The diagnostic utility of pleural markers for tuberculosis pleural effusion. Ann Transl Med 2020;8(9):607. doi: 10.21037/ atm.2019.09.110 interleukin 2 receptor in pleural fluid. Int J Tuberc Lung Dis 2000;4:975-9.

85. Harita S, Nogami N, Kikuchi T, et al. Preliminary evaluation of soluble IL-2 receptor and type III procollagen $\mathrm{N}$-terminal aminopeptide in pleural fluid for differentiating tuberculous, carcinomatous and parapneumonic pleural effusions. Respirology 2002;7:311-5.

86. Aoe K, Hiraki A, Murakami T, et al. Diagnostic Significance of Interferon- $\gamma$ in Tuberculous Pleural Effusionsa. Chest 2003;123:740-4.

87. Hiraki A, Aoe K, Eda R, et al. Comparison of Six Biological Markers for the Diagnosis of Tuberculous Pleuritis. Chest 2004;125:987-9.

88. Tural Önür S, Sökücü SN, Dalar L, et al. Are soluble IL-2 receptor and IL-12p40 levels useful markers for diagnosis of tuberculous pleurisy? Infect Dis (Lond) 2015;47:150-5. 